\title{
Automated Detection and Quantification of Prostate Cancer in Needle Biopsies by Digital Image Analysis
}

\author{
Vamsi Parimi ${ }^{1}$, Laurie J. Eisengart ${ }^{2}$, Ximing J. Yang1,2* \\ ${ }^{1}$ Pathology Core Facility, Robert H. Lurie Comprehensive Cancer Center, Chicago, IL, USA \\ ${ }^{2}$ Department of Pathology, Northwestern University, Chicago, IL, USA \\ Email: xyang@northwestern.edu
}

Received 3 June 2014; revised 30 June 2014; accepted 12 July 2014

Copyright (C) 2014 by authors and Scientific Research Publishing Inc.

This work is licensed under the Creative Commons Attribution International License (CC BY). http://creativecommons.org/licenses/by/4.0/

(c) (i) Open Access

\begin{abstract}
Introduction: Triple immunohistochemical (IHC) stains including antibodies specific for alphamethylacyl-CoA-racemase and basal cell markers have been a valuable aid in accurate identification of prostate carcinoma. However, accurate quantification of minuscule areas of prostate carcinoma in biopsy specimens can often be a challenge. Here we assessed the diagnostic value and quantitative use of automated digital image analysis on triple IHC stained prostate needle biopsies. Methods: Twelve cases of prostate needle biopsy material including 75 needle cores were stained with triple-antibody cocktail (P504S + 34 BE12 + p63). Slides were digitally scanned with the APERIO digital image analyzer and evaluated with the GENIE pattern and color recognition digital image analysis that we developed. A slide with known areas of adenocarcinoma, high grade prostatic intraepithelial neoplasia (PIN), benign glands and stroma was used as a training set for the automated digital image analysis platform. Results: Among 75 needle biopsy cores, 19 (25.33\%) contained adenocarcinoma by histology. Digital image analysis recognized adenocarcinoma in $95 \%$ of these needle biopsies. The average area of the needle biopsy was $7.63 \mathrm{~mm}^{2}$ and overall the average area of tumor was $0.196 \mathrm{~mm}^{2}$. The smallest area of tumor recognized by the program was $0.0022 \mathrm{~mm}^{2}\left(0.0363 \%\right.$ of the core) and the largest was $0.62 \mathrm{~mm}^{2}(8.17 \%$ of the core) among needle core biopsies. False positives resulted from areas of high grade PIN with patchy basal cells. The false negative was caused by uneven AMACR staining in one area of adenocarcinoma. Digital recognition of areas of interest was improved by three successive image analysis training which increased the sensitivity and specificity from $83 \%$ and $89 \%$ to $90 \%$ and $93 \%$, respectively. Conclusions: Digital image analysis in concert with IHC triple staining may be useful for accurate detection and quantitative analysis of small foci of prostatic adenocarcinoma. Defining methods to increase the sensitivity and specificity of quantitative automated digital image analysis will likely evolve as an area of investigation. Future automated digital scanning and innovative pattern and
\end{abstract}

*Corresponding author.

How to cite this paper: Parimi, V., Eisengart, L.J. and Yang, X.J. (2014) Automated Detection and Quantification of Prostate Cancer in Needle Biopsies by Digital Image Analysis. Open Journal of Pathology, 4, 138-150.

http://dx.doi.org/10.4236/ojpathology.2014.43020 
color recognition technologies may open avenues for classifying a variety of prostate lesions.

\section{Keywords} Prostatic Adenocarcinoma, Needle Biopsy, Alpha-Methylacyl-CoA-Racemase, P504S, Basal Cell,
Immunohistochemistry, Automated Digital Image Analysis

\section{Introduction}

Each year, more than a million of American men will undergo prostate biopsy after clinical and serological prostate specific antigen (PSA) screening [1]. Based on the recent global cancer statistics estimates, the global incidence of prostatic adenocarcinoma is expected to double by 2030 [2]. The emphasis on early prostatic adenocarcinoma detection has led to mass screening of men leading to small cancer foci detection on prostate needle core biopsy specimens [1] [3]-[6]. Conventional manual bright-field microscopic examination of transrectal ultrasonography (TRUS)-guided prostate needle core biopsy is the gold standard for diagnosis of prostatic adenocarcinoma [7] [8]. However, manual diagnoses depend heavily on the expertise and experience of the genitourinary pathologists, giving it poor intra- and inter-observer reliability. Further, manual microscopic examination is time consuming, particularly since each biopsy specimen typically contains 12 cores of prostatic tissue.

Accurate classification of prostatic tissue is critical because of the risks associated with incorrect diagnostic results. The small foci of prostatic adenocarcinoma in needle biopsy specimens are both heterogeneous and multifocal, and correct identification of foci is one of the major clinical challenges in genitourinary pathology. On H\&E stained (Figure 1) sections, the primary criteria for identification of prostatic adenocarcinoma include acinar proliferation, nuclear atypia and nucleolar prominence, absence of acinar basal cell lining together with presence of characteristic extracellular material in malignant glands [3] [9] [10]. In up to $9.0 \%$ of all biopsies,

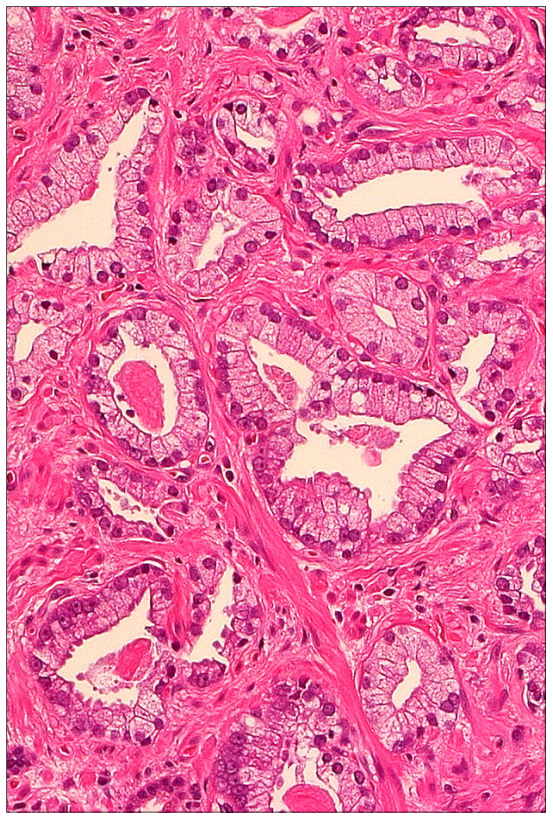

(a)

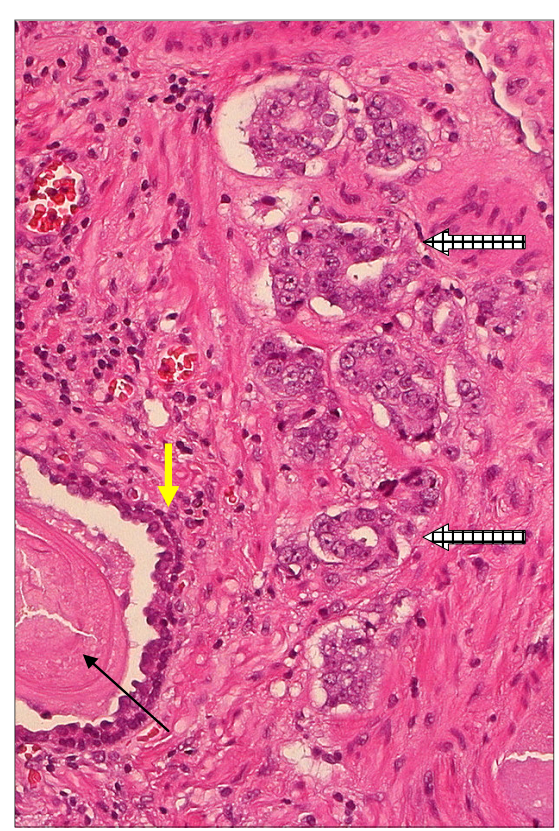

(b)

Figure 1. Hematoxylin and Eosin staining of prostatic adenocarcinoma. (a) Gleason $3+$ 3 prostatic adenocarcinoma. (b) Gleason $4+4$ prostatic adenocarcinoma with adjacent benign gland showing acinar basal cell lining (yellow arrow) and Corpora amylacea (black arrow). Note acinar proliferation, nuclear atypia and nucleolar prominence, absence of acinar basal cell lining together with presence of characteristic extracellular material surrounding malignant glands (grid arrows). Original magnifications: $\times 200$ (a) (b). 
small acinar proliferation falls below manual diagnostic threshold on hematoxilin and eosin (H\&E) staining [11] and $59 \%$ of these cases of missed proliferation turn out to be malignant after using supplementary immunohistochemical staining [12] [13].

No single morphologic feature can account reliably by itself in the diagnostic process. So an obvious diagnosis of malignancy is difficult, especially in limited foci or in small atypical acinar lesions in needle core biopsy specimens. With its varied morphological patterns, prostatic adenocarcinoma can be confused with several histological patterns like atrophy, hyperplasia and prostatic intraepithelial neoplasia and processes like seminal vesicle, inflammatory and reactive conditions in thin core needle biopsies [14]. False positive prostatic adenocarcinoma diagnosis albeit uncommon, may lead to unnecessary prostatectomies, adjuvant therapies and medico-legal consequences [15]. The greatest potential risk of prostate biopsyis that a prostatic adenocarcinoma may be missed because of sampling reasons, or under recognized by pathologists. Underdiagnoses (false negativity) may delay early diagnosis and treatment. Thus accuracy of pathology diagnosis may be improved by application of reliable and objective tumor specific markers for prostate adenocarcinoma [16].

In the normal and pre-invasive human prostate glandular tissues, the luminal columnar secretory epithelium is separated from the basement membrane and stroma by flattened basal cells. Basal cell layer is typically quite inconspicuous and may be hard to detect on routine H\&E stained sections (Figure 1). Immunohistochemical staining (Figure 2) for basal cell specific high-molecular-weight cytokeratin (34/E12, cytokeratin 5/6), bcl-2 or for p63 antibody shows the basal cell envelop to be complete even where no basal cells are recognized with routine H\&E stains [17]-[21]. The identification of basal cells of prostate glands signifies benign glands [17] [19] [21] [23] [24], which are consistently absent in invasive prostatic adenocarcinoma [22]. However, assessment of the basal layer only has several pitfalls like uneven basal layer staining and false negative staining due to prolonged fixation [25]. And also, several benign mimickers of prostatic adenocarcinoma, including atrophy, atypical adenomatous hyperplasia (AAH), nephrogenic adenoma, and mesonephric hyperplasia, can stain negatively with basal cell layer markers, and thus, a negative basal cell marker immunostain alone does not exclude a diagnosis of benignancy [21].

Another important consideration in the diagnosis of prostatic cancer is the selection of highly sensitive and specific biomarker. Of several prostatic adenocarcinoma specific biomarkers, AMACR is prominent. The sensitivity and specificity of AMACR in the detection of prostate cancer is $97 \%$ and $100 \%$ respectively [26]. Alpha-methylacyl-CoA racemase-P504S (AMACR) was discovered by Xu J. et al. [27] using cDNA library subtraction and later validated as a promising specific marker for prostatic adenocarcinoma by several groups [16] [26] [28]-[35]. AMACR was described as a gene playing a critical role in peroxisomal $\beta$ oxidation of branched chain fatty acid molecules [36]. The predominately cytoplasmic localization expression of AMACR is consistently up-regulated in prostatic adenocarcinoma and precursor lesion (high-grade prostatic intraepithelial neoplasia-PIN), is absent in the vast majority of benign prostate tissue [27]. High grade PIN in prostate needle biopsy specimen is clinically significant because the risk of carcinoma on biopsy range from 27\% to 79\% [37]-[44]. AMACR expression can also be identified in prostatic atrophy, atypical adenomatous hyperplasia and benign prostatic glands, and accordingly, a diagnosis of prostatic adenocarcinoma should not be based solely on a

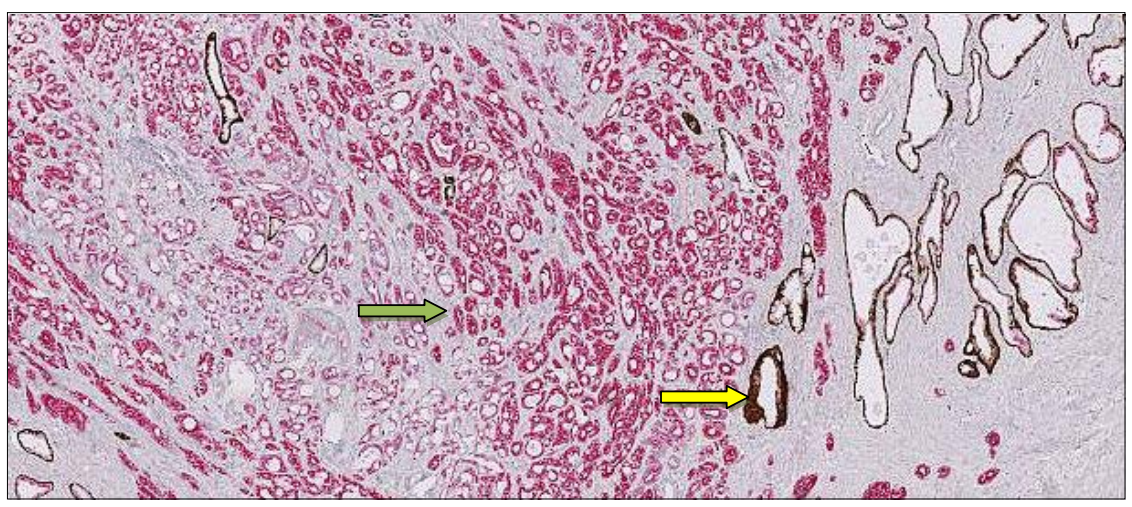

Figure 2. Differential cocktail immunostaining representing prostatic adenocarcinoma; AMACR positive and keratin negative (green arrow) and benign prostate glands; Keratin positive and AMACR negative (yellow arrow). Original magnifications: $\times 40$. 
positive AMACR immunostain, especially when the luminal staining is weak and/or noncircumferential [30] [33]. In that way Alpha-methylacyl-coenzyme-A racemase (AMACR) is a sensitive marker of prostatic adenocarcinoma (except for atrophic, foamy gland, and pseudohyperplastic variants), and its detection by immunohistochemical staining in atypical prostatic lesions can be very useful in confirming a diagnosis of prostatic adenocarcinoma [45]. So a mutually exclusive staining of AMACR (positivity) and negative basal cell marker reactions are useful clinically to confirm the presence of residual prostatic adenocarcinoma after hormonal or radiation therapy [30] [46]-[48].

Manual evaluation of prostate histologic biopsy sections is time consuming and highly dependent on user's experience, resulting in inter and intra observer variability [49]. The improvement in digital technology and virtual storage capabilities has led to the discovery of whole slide digital scanners providing a method for image analytical software to handle complex morphocolorimetric tasks on histological images. Automated microscopy and computerized processing have made strides in digital pathology, which has increased accuracy for tissue based detection and quantification thereby minimizing observer variability. Several groups have proposed quantitative automated digital image analysis for prostatic adenocarcinoma diagnosis and Gleason grading using digitized H\&E microscopic images. These studies typically ranged group classification rate between $72 \%$ and 88\% [50] [51]. The limitations to these studies include diverse and high complexity of histology traits used on hematoxylin and eosin stained slides and subjective image analysis evaluation. The aim of our paper is to investigate the feasibility for a high throughput automation utilizing color and pattern recognition software to identify and quantify areas of interest among diagnostic slides stained with triple stain (AMACR + 34 $\beta E 12+$ p63) with increased reproducibility and predictive accuracy. Our study focused on total surface area of biopsy tissue, benign prostate tissue, areas of prostate intraepithelial neoplasia (PIN) and invasive prostatic adenocarcinoma on the whole slide.

\section{Materials and Methods}

\subsection{Case Selection}

Prostatic adenocarcinoma needle core biopsies for IHC analysis were obtained from 12 patients with abnormal blood PSA (level $\geq 4.0 \mathrm{ng} / \mathrm{ml}$ ) and/or an abnormal finding during a digital rectal examination at Northwestern Memorial Hospital from 2009 to 2010. Specimens were obtained from the operating room immediately after ultrasound guided biopsy. The criterion standard for accurately diagnosing prostatic adenocarcinoma was reviewed by one of two experienced genitourinary pathologists (X.J.Y. and L.E.) using a combination of routine hematoxylin-eosin stained slides. All diagnoses made on needle biopsy specimens were confirmed by pathologic review on prostatectomy resection specimens. Benign prostate tissue, prostate intraepithelial neoplasia, mimickers of (Prostatic adenocarcinoma) along with Gleason grades higher than $4+4$ were used as test controls during the evaluation process [52]. All the case and control slides were de-identified of case information and the study was approved by institutional research committee.

\subsection{Immunohistochemistry}

Immunohistochemistry was carried out using the ready to use EnVision ${ }^{\mathrm{TM}}$ DuoFLEX IC004kit (DAKO Corp., Carpinteria, CA) and Dakoautostainer. DuoFLEX cocktail contains monoclonal rabbit anti-human AMACR, clone 13H4, monoclonal mouse anti-human cytokeratin, high molecular weight (48-67 kDa), clone 34ßE12 and monoclonal mouse anti-human cytokeratin 5/6 (58 kDa), cloneD5/16 B4 (Link). The visualization is based on peroxidase (HRP) using DAB+ as chromogen for $34 \beta \mathrm{E} 12$ + CK 5/6 and alkaline phosphatase (AP) using Permanent Red as chromogen for AMACR. The procedure is a simultaneous double staining where two antigens are visualized using HRP/DAB+ and AP/Permanent Red, respectively. The reaction is visualized by EnVision ${ }^{\mathrm{TM}}$ DuoFLEX DAB+ Chromogen for mouse antibody and EnVision ${ }^{\mathrm{TM}}$ DuoFLEX Permanent Red for rabbit antibody. The system is biotin-free, thus significantly reducing non-specific staining resulting from endogenous avidin-biotin activity. Briefly deparaffinized slides were hydrated then placed in citrate buffer (pH 6.0) and steamed for 14 minutes. Endogenous peroxidase activity was quenched by incubation with DAKO peroxidase block for $5 \mathrm{~min}$ at room temperature. Slides were then washed and incubated with primary antibody (1:16,000 dilution of antiserum) overnight at $4^{\circ} \mathrm{C}$. Secondary anti-rabbit antibody-coated polymer peroxidase complex was applied for $30 \mathrm{~min}$ at room temperature. Substrate/chromogen was applied and incubated for 5 - $10 \mathrm{~min}$ at room 
temperature. Slides were counterstained with hematoxylin.

To evaluate the usefulness of automated digital recognition and quantification of AMACR expression, twelve 18-gauge prostate needle biopsy sections composed of 75 needle cores with adenocarcinoma were stained with triple-antibody cocktail (AMACR + 34/E12 + CK 5/6). The diagnosis of prostatic adenocarcinoma was established from examination of H\&E stained sections by at least 2 surgical pathologists (X.Y. and L.J.E.) and was confirmed by absence of basal cell staining and/or a positive result for AMACR. Positive AMACR staining was defined as continuous dark cytoplasmic staining or apical granular staining patterns in cells, which could be easily observed at low-power magnification (X100). Benign epithelial glands and prostate cancer cells were scored for AMACR staining intensity on four tiered system ranging from negative to strong expression [53].

\subsection{Automated Digital Analysis}

$12 \mathrm{H} \& \mathrm{E}$ and corresponding triple stained de-identified glass slides were digitally scanned with the Aperio® ScanScope ${ }^{\circledR}$ XT slide scannerand digital image analyzer (Aperio Technologies, Inc., Vista, CA, USA) at 20X objective with a resolution of $0.5 \mu \mathrm{m} /$ pixel using a $\times 20 / 0.75$ NA Plan Apo objective. The digital images were captured in single planes without Z-stacking or multi-plane imaging. The images were viewed using Spectrum ${ }^{\mathrm{TM}}$ and Imagescope ${ }^{\mathrm{TM}}$ software (Aperio Technologies Inc., Vista, CA, USA)and evaluated with GENIE (Aperio Technologies, Inc.Vista, CA) pattern and color recognition software. A prostatectomy slide stained with triple antibody (34/E12 + CK 5/6 + AMACR) cocktail with known areas of adenocarcinoma, high grade prostate intraepithelial neoplasia (PIN), benign glands and stroma was used for defining areas of interest and as a training set for the automated digital image analysis development. Qualitative region selection, region boundaries and digital image analysis performance thresholds was determined by pathologists. Software's training set and pattern classification technique was created from typical areas on a control slide. Areas of classes (Figure 3) were then defined with color codes (Table 1) and a montage was created to run the training image analysis (classifier). In order to determine the accuracy of detection of respective components on the glass slide and region quantification, classifier was used to test the control set of slides (Figure 4). Performance characteristics of classifier are evaluated in terms of sensitivity and specificity of class detection and ensuing area quantification per slide. The optimization of the automated digital image analysis together with progressive iterations were verified by reference pathologist specialized in prostate pathology. Once the best achievable performance of the classifier was reached using the training set, we analyzed the prostatic biopsy samples. Biopsy slides were evaluated for total of three times with successive improvements of the Genie classifier. The sensitivity and specificity for the current study's digital recognition software was set to $90 \%$ and $93 \%$, respectively, due to practical considerations.

All statistical values were determined with Microsoft Excel. These values included percent values and mean

Table 1. Class A: Prostatic adenocarcinoma has been defined when AMACR staining (intensity scale 1,2 or 3 ) positive and $34 \beta E 12+$ CK $5 / 6$ is staining negative in small circular prostate glands with small to no lumen. Class B: High-grade PIN has been classified when AMACR staining (intensity scale 1, 2 or 3) positive along with $34 \beta E 12+$ CK 5/6 expression. Class C: Benign prostate glands are categorized when AMACR staining negative and (intensity scale 0 ) and with $34 \beta \mathrm{E} 12+\mathrm{CK} 5 / 6$ expression. Class D: Stroma of prostate gland. Class E: Glass background. For every class, three unique areas of respective morphological patterns were sampled for the automated digital image analysis training.

\begin{tabular}{ccc}
\hline Class & Color Code & Classifier Designee \\
\hline A & Red & Prostatic adenocarcinoma \\
B & Yellow & PIN \\
C & Green & Benign Prostate \\
D & Blue & Stroma \\
E & Pink & Glass \\
\hline
\end{tabular}




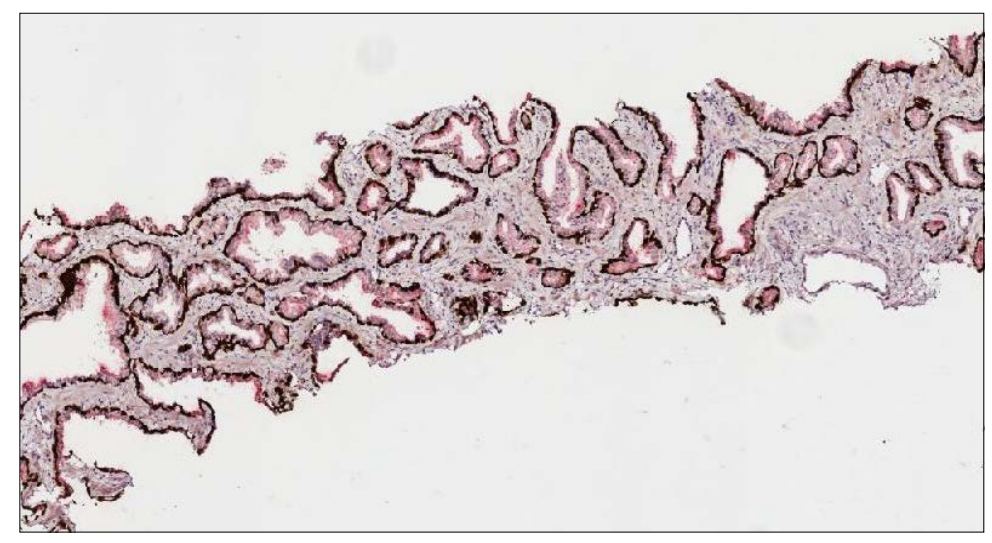

(a)

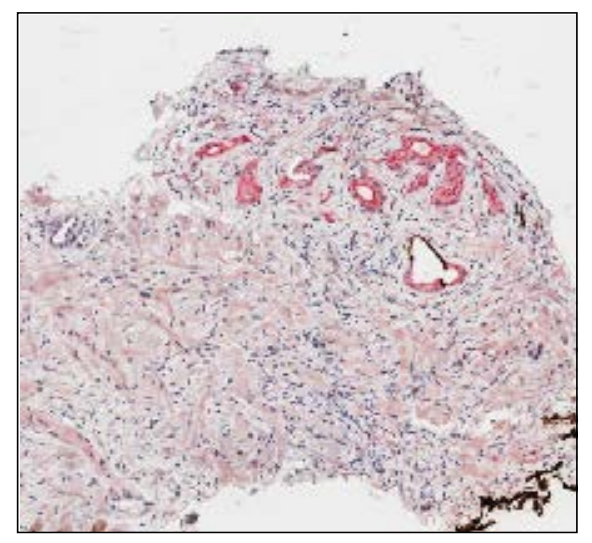

(b)

Figure 3. Triple cocktail immunostaining (34/ E12 + CK 5/6 + AMACR) in prostate needle core biopsy: (a) AMACR showed weak epithelial staining pattern in benign glands also. Linear basal staining with keratins (34 $\beta$ E12 and CK 5/6) is evident. (b) Microscopic foci of prostatic adenocarcinoma with intensive AMACR staining without basal keratin staining. Original magnifications: $\times 40$.

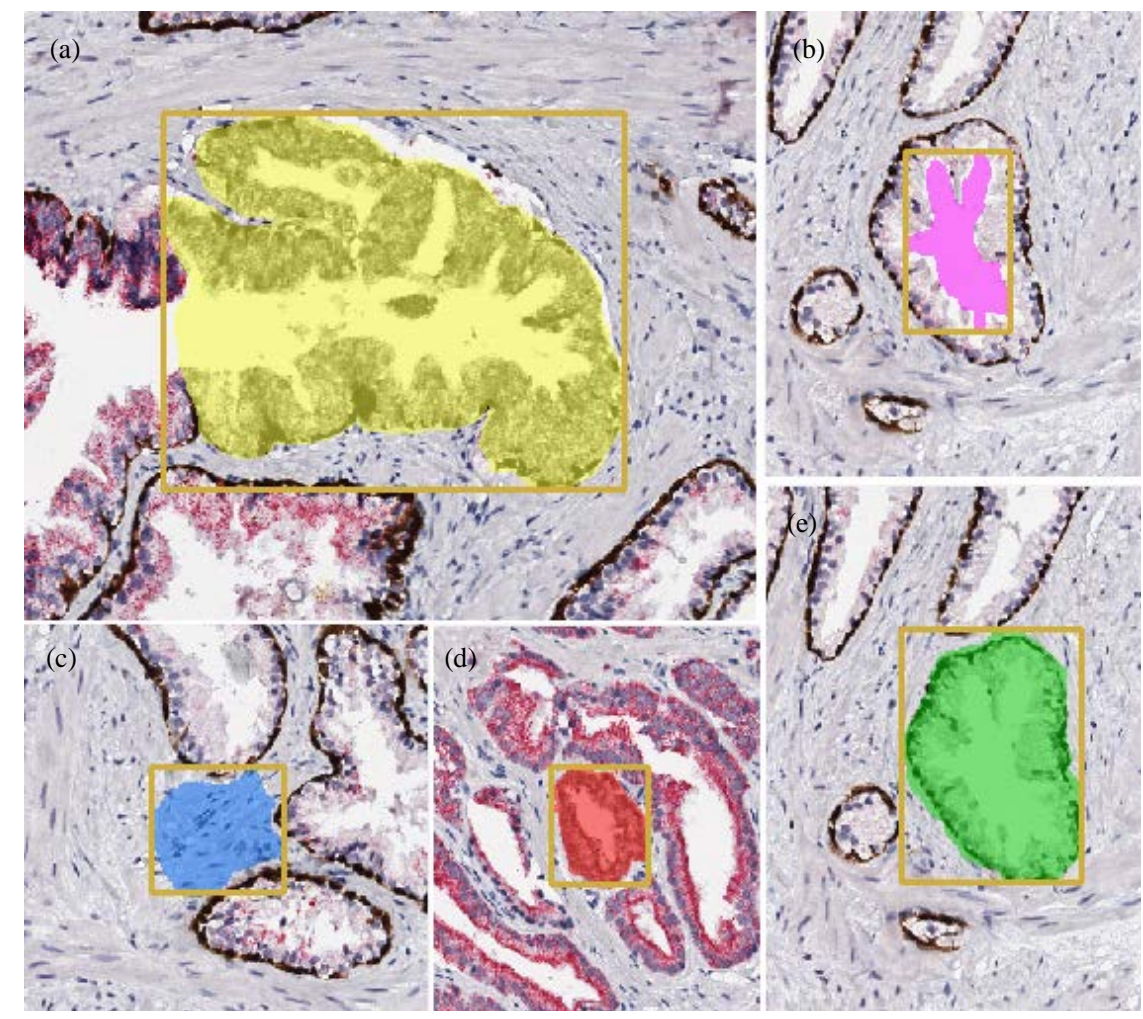

Figure 4. Prostate biopsy montage with the class definition sets. Class A: Prostatic adenocarcinoma has been defined when AMACR staining (intensity scale 1,2 or 3 ) positive and 34 $\beta$ E12 + CK 5/6 is staining negative in small circular prostate glands with small to no lumen. Class B: PIN has been classified when AMACR staining (intensity scale 1,2 or 3 ) positive along with $34 \beta \mathrm{E} 12+\mathrm{CK} 5 / 6$ expression. Class C: Benign prostate glands are categorized when AMACR staining negative and (intensity scale 0 ) and with $34 \beta$ E12 + CK 5/6 expression. Class D: Stroma of prostate gland. Class E: Glass background. Areas of Classes were then defined with color codes and a montage is created to run the training analysis (classifier). (a) PIN (yellow); (b) glass background (pink); (c) stroma (blue); (d) carcinoma (red) and (e) benign glands (green). Original magnifications: $\times 200$. 
values. These values were used to evaluate the percent of correct identification on H\&E glass slides by expert pathologists (X.Y. and L.E.) compared with digital slides classified by automated digital image analysis.

\section{Results}

Manual diagnostic evaluation of the prostate needle core biopsies resulted in Gleason scores [54]-[56] ranging from 5 to 9. Near uniform moderate-to-strong AMACR protein expression (score $=3$ and 4 , respectively) was observed in prostatic adenocarcinoma samples from men with clinically localized prostatic adenocarcinoma (Figure 5). Adjacent areas of benign prostate tissue did not express AMACR, verifying that AMACR a highly specific prostatic adenocarcinoma marker. We compared the results of automated recognition to the AMACR, 34 $\beta$ E12 and CK 5/6 staining manually and determined little difference in accuracy between methods. Among 75 needle biopsy cores, 19 (25.33\%) contained prostatic adenocarcinoma by histology and triple stain; digital image analysis recognized prostatic adenocarcinoma in $95 \%$ of all needle biopsies (Figure 6). The average area of the needle biopsy was $7.63 \mathrm{~mm}^{2}$. Overall, the average area of tumor was $0.196 \mathrm{~mm}^{2}$. The smallest area of tumor recognized by the program was $0.0022 \mathrm{~mm}^{2}\left(0.0363 \%\right.$ of the core) and the largest was $0.62 \mathrm{~mm}^{2}(8.17 \%$ of the core). We re-reviewed all cases with discordant assignment. We found false positives (Figure 7) resulted from areas of high grade PIN with patchy basal cells. The false negative was caused by uneven AMACR staining in one area of Prostatic adenocarcinoma (Figure 8). Higher Gleason score histology with fused glands and scattered individual cancer cells with complex structures were also positively identified by the automated digital image analysis.

Digital recognition of areas of interest was improved by three successive digital image analysis trainings which increased the sensitivity and specificity from $83 \%$ and $89 \%$ respectively. The application of the 4 - 6 characteristic histologic features to each class definition reduced the number of overlapping detections to a single consistent detection during multiple recognition runs. This sequential testing method also reduced the number of false positives. We have also observed a tradeoff between probability of true detection and false detections on varying classifier threshold. Development of Genie classifier for triple immunostained slides took nearly 3 hours. The training process took around $40 \mathrm{~min}$ and testing analysis's detecting capabilities took approximately $10 \mathrm{~min}$ per image running on a conventional desktop (2GB RAM, Dual Core CPU, $2 \mathrm{GHz}$ of processing speed). The image analysis and processing time depended on surface area of initial specimen.

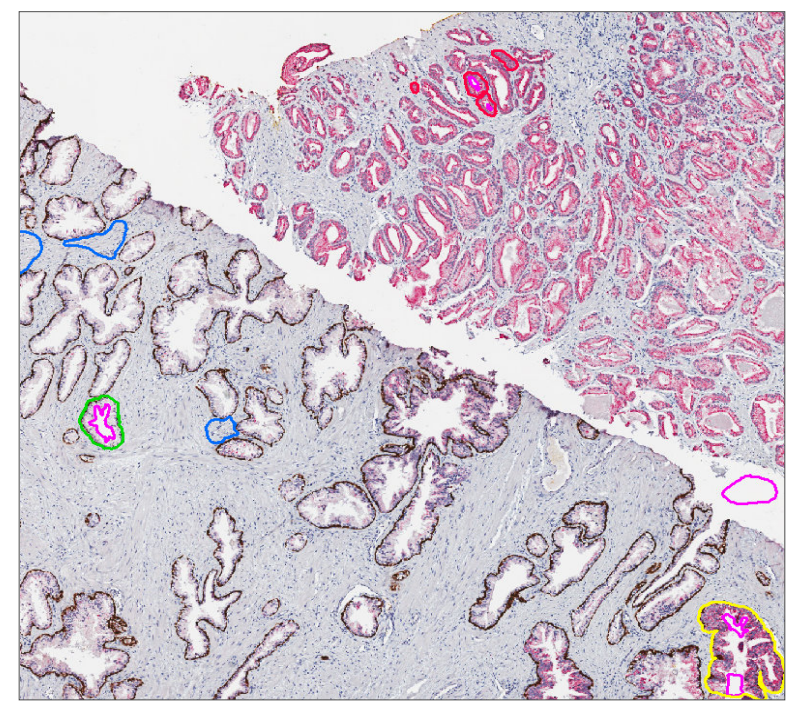

(a)

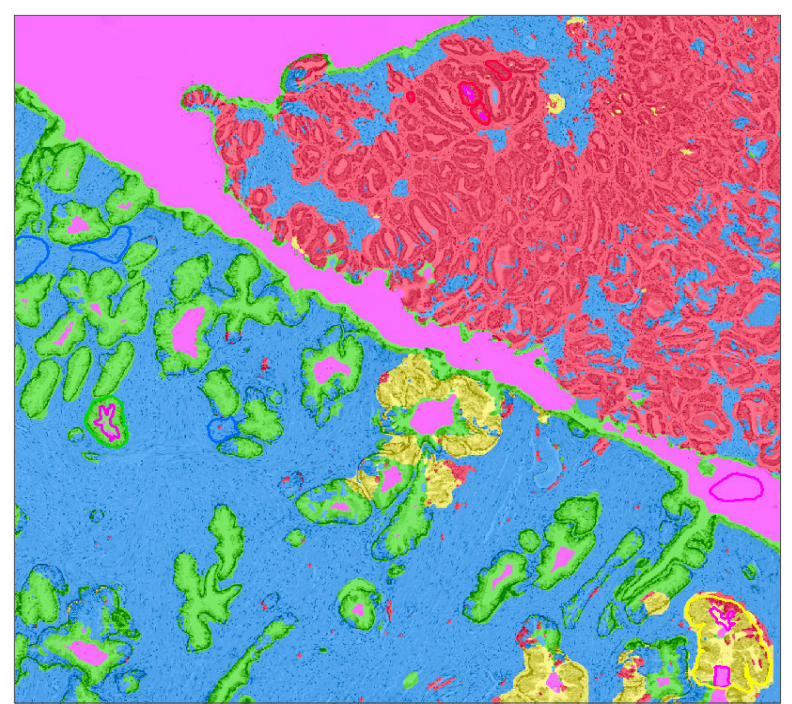

(b)

Figure 5. Genie montage sampling and classifier overlay in a prostatectomy control specimen: (a) Triple Stain IHC: AMACR showed weak staining pattern in benign prostatic glands and continuous basal staining with 34 $\beta$ E12 and CK 5/6. Homogeneous circumferential AMACR expression without basal cell staining in prostatic adenocarcinoma. (b) Prostatic glands with high intensity AMACR expression with absent basal cell marker expression are precisely recognized as prostatic carcinoma. Stroma (blue), benign glands (green), PIN (yellow), carcinoma (red), and glass background (Pink) classifications are represented in respective color codes. Original magnifications: $\times 40$. 


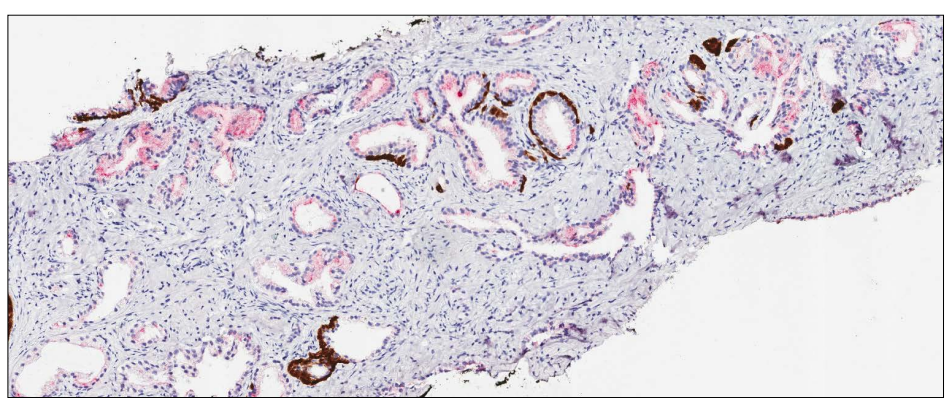

(a)

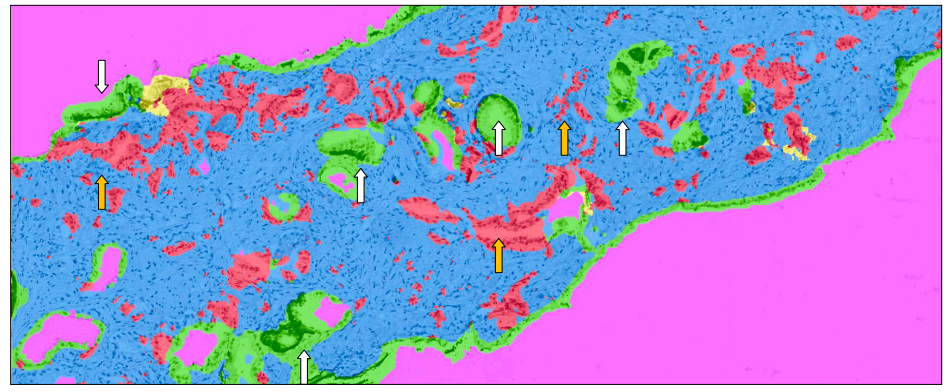

(b)

Figure 6. Example of false positive identification of prostatic adenocarcinoma. (a) Triple immunostain: AMACR showed widespread weak to moderate epithelial staining pattern in benign. Pathologist diagnosed it as partial atrophy of prostate glands on needle biopsy. Patchy circumferential basal staining with $34 \beta E 12$ and CK 5/6 is evident. (b) Automated color and pattern recognition classifier overlay: Moderately intense AMACR staining without basal cell marker expression is misrecognized as prostate carcinoma (orange arrows). Area of atrophic glands with basal cell expression is appropriately recognized as benign glands (white arrows). Original magnifications: $\times 100$.

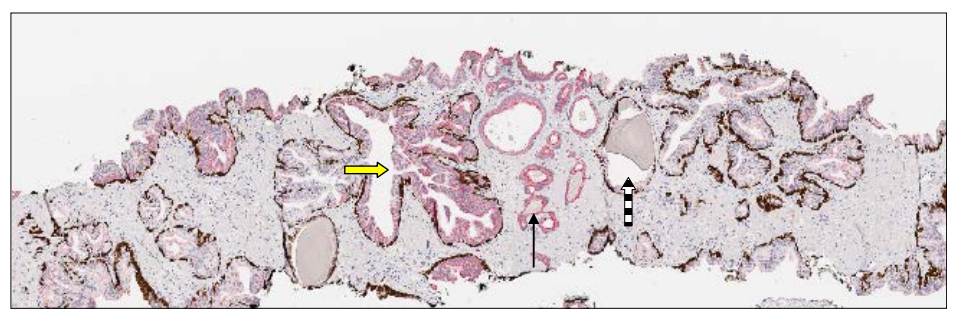

(a)

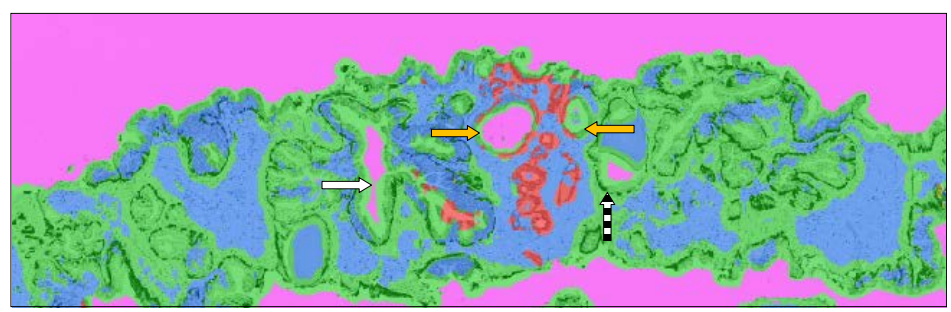

(b)

Figure 7. Immunohistochemical staining of AMACR, 34/E12 and CK 5/6 and automated color and pattern recognition image analysis overlay in adenocarcinoma (narrow solid black arrow) of prostate needle biopsy: (a) Triple immunostain: AMACR showed widespread weak to moderate epithelial staining pattern in benign atrophic glands and intensive expression in PIN and adenocarcinoma (black arrow). Patchy unremarkable basal staining with $34 \beta \mathrm{E} 12$ and CK $5 / 6$ is evident in high-grade PIN lesion (yellow arrow). Corpora amylacea (striped arrow). (b) Classifier overlay: Absence of AMACR staining has always been recognized as a benign area; Corpora amylacea (striped arrow). High-grade PIN lesion recognition and subsequent detection had failed in almost $50 \%$ of the areas (white arrow). Large low grade prostatic adenocarcinoma lesion was recognized as benign entity owing to the morphological criteria (orange arrows) of the classifier. Original magnifications: $\times 100$. 


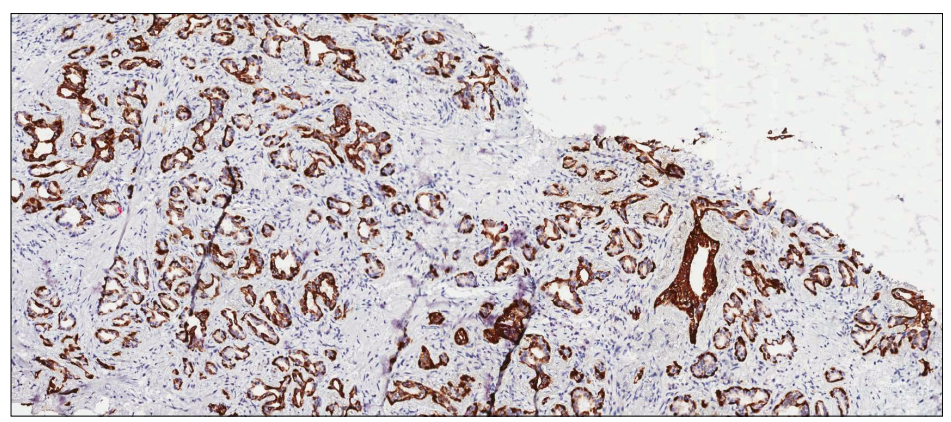

(a)

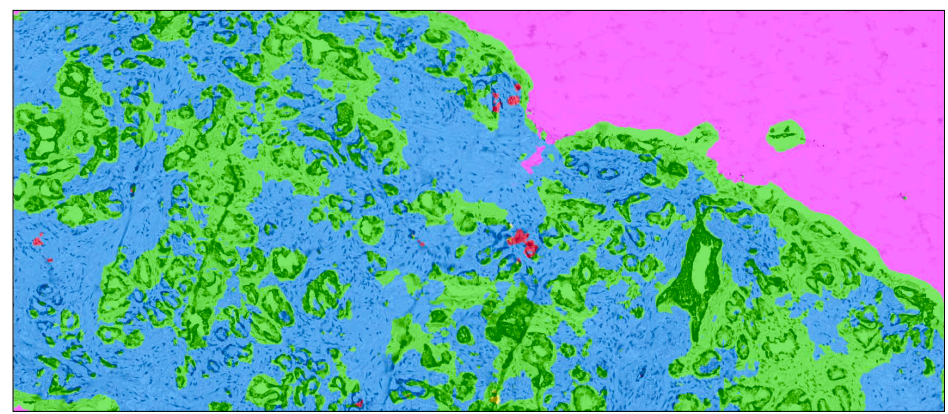

(b)

Figure 8. Classifier performance of atypical adenomatous hyperplasia (adenosis) in prostate needle biopsy. (a) Tripleimmunostain: Absence of AMACR staining in benign adenomatous glands. Note the circumferential basal staining of $34 \beta \mathrm{E} 12$ and CK 5/6. (b) Classifier overlay: Absence of AMACR staining has always been recognized as a benign area and patchy AMACR staining was recognized as false positive for prostate adenocarcinoma (red). Original magnifications: $\times 100$.

\section{Discussion}

We found that the automated recognition tool was capable of detecting tissue pathologies with a comparable level of accuracy, demonstrating its feasibility as a cancer detection tool. The current study investigates the possibility of an automated recognition tool in identifying and quantifying various prostate pathologies differentiated by triple staining (AMACR, 34/E12 and CK 5/6). Our work is novel with regards to multiple aspects: to our knowledge it is the first time that GENIE classifier is applied to triple staining immunohistochemistry for prostate pathology. Furthermore, our automated imaging analysis method can be used for the quantification of prostatic adenocarcinoma and PIN lesions in needle biopsy and radical prostate resections in a time frame of 10 minutes. We believe this as a fundamental limitation of processing power of the computer and can be vastly improved due to current technological advancementsin computer hardware.

Prostatic adenocarcinoma is a heterogeneous disease and we have accounted for this variability by incorporating samples with various Gleason scores. Irrespective of Gleason score, the automated digital image analysis appropriately detected prostatic adenocarcinoma in all the biopsy specimens in which AMACR staining was present. Although the antibodies used were validated internally, our study is subject to a number of limitations. Perhaps, the most significant is the classifier errors principally depended upon initial staining characteristics of Prostatic adenocarcinoma. Focal basal cell staining loss was misrepresented as prostate adenocarcinoma in benign and high grade PIN lesions. Partial atrophy region with large glands, absent basal cell staining and AMACR positive areas were designated as prostatic adenocarcinoma. Immune staining physiognomies (Figure 9) were identified as a leading cause of false positivity of prostate adenocarcinoma while running the automated detection image analysis.

Our method may be used to enhance the detection of prostatic adenocarcinoma or quantitative measurement of tumor volume in pathology clinical practice, similar to the computer assisted diagnosis (CAD) currently used in radiology practice. We also believe in its potential utility for other pathology assessments during pathology central review process and in research for high through put image analysis for scientific data capture and evaluation. It is our future objective to study prognostic clinical outcomes based on computational quantification of 


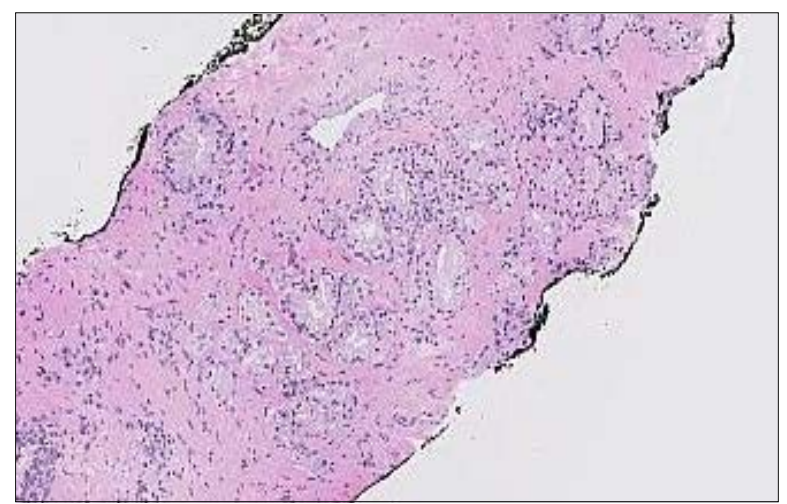

(a)

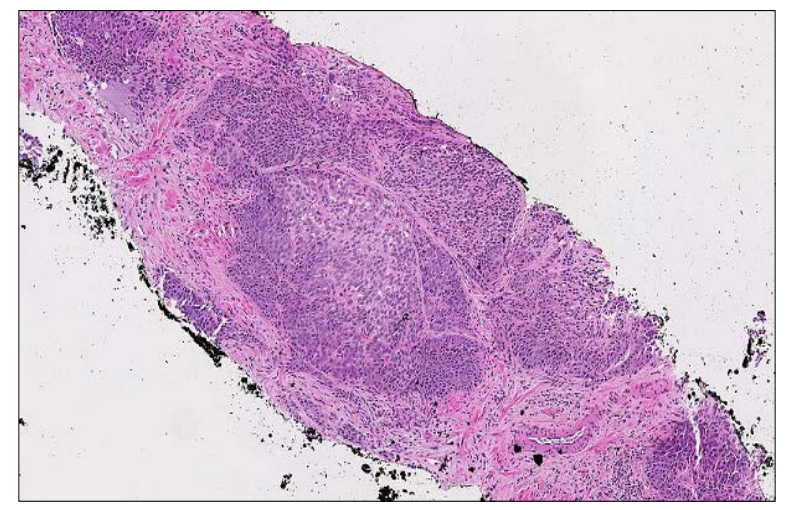

(c)

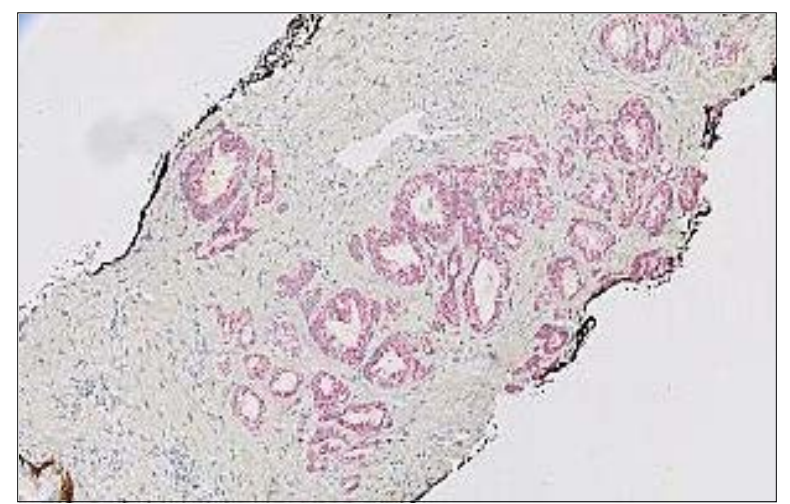

(b)

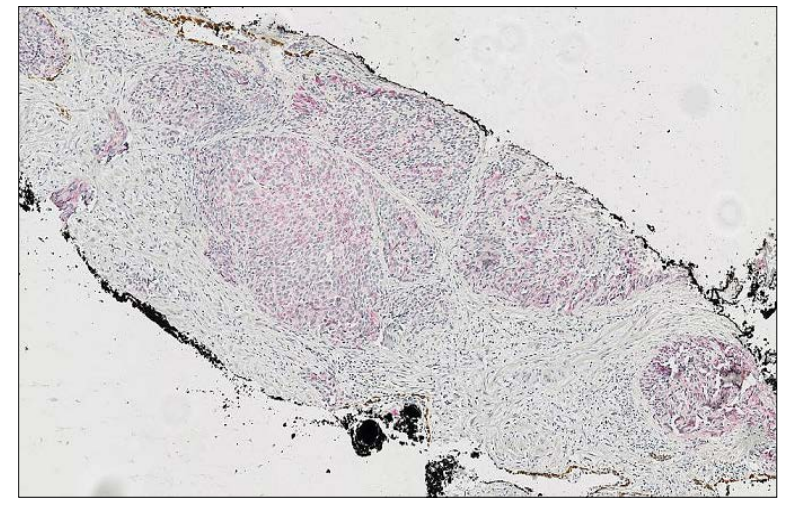

(d)

Figure 9. Illustration of cases in which P504S (AMACR) exhibited variable staining intensities. (a) and (b): H\&E of prostatic adenocarcinoma Gleason grade $(3+3)$ and triple stain with high intensity AMACR staining and Gleason grade $(5+5)$ H\&E and triple stain with low intensity AMACR staining. Original magnifications: ×100 (a), (b); ×40 (c), (d).

prostatic adenocarcinoma among prostate needle core biopsies in a large cohort of cases. This study is a proof of principle and therefore performed on limited sample number from a single institution. These results must be further validated across independent institutions and greater sample size.

\section{Conclusion}

Currently limitations of imaging analysis applied in pathology clinical practice include a lack of standardized quantitative imaging tools; inability to reproduce standard automated digital image analysis across institutions, lack of generic image analysis validation process and deficiency of integrative platforms [49]. Histological evaluation of the prostate needle core biopsy material remains a main challenge in pathology practice. It has been shown previously by our group and other investigators that triple immunostains including AMACR and basal cell marker (34 $\beta$ E12 and CK 5/6) are helpful in establishing the diagnosis of prostatic adenocarcinoma [16] [28] [29] [57] [58]. In this study, we used automatic imaging analysis of triple immunostained slides and concluded that this is sensitive and reliable method for detecting prostatic adenocarcinoma. The current study provides a basis for potential utility of automated systems for qualitative analyses of prostate needle core specimens in clinical and research settings. Our feasibility study demonstrates a novel approach that may aid pathologists in the implementation of automated image analysis in the field of prostatic adenocarcinoma diagnostics.

\section{Acknowledgments}

We thank Steve Clapp of Aperio for technical support. This work was supported by Prostatic adenocarcinoma SPORE grant (CA90386). Finally, we would like to thank Erin Boutwell and anonymous reviewers for their insightful comments and helpful suggestions. 


\section{References}

[1] DiGiuseppe, J.A., Sauvageot, J. and Epstein, J.I. (1997) Increasing Incidence of Minimal Residual Cancer in Radical Prostatectomy Specimens. American Journal of Surgical Pathology, 21, 174-178. http://dx.doi.org/10.1097/00000478-199702000-00006

[2] Jemal, A., et al. (2011) Global Cancer Statistics. CA: A Cancer Journal for Clinicians, 61, 69-90. http://dx.doi.org/10.3322/caac.20107

[3] Epstein, J.I. (1995) Diagnostic Criteria of Limited Adenocarcinoma of the Prostate on Needle Biopsy. Human Pathology, 26, 223-229. http://dx.doi.org/10.1016/0046-8177(95)90041-1

[4] Epstein, J.I. and Potter, S.R. (2001) The Pathological Interpretation and Significance of Prostate Needle Biopsy Findings: Implications and Current Controversies. Journal of Urology, 166, 402-410. http://dx.doi.org/10.1016/S0022-5347(05)65953-8

[5] Epstein, J.I., Walsh, P.C., Carmichael, M. and Brendler, C.B. (1994) Pathologic and Clinical Findings to Predict Tumor Extent of Nonpalpable (Stage T1c) Prostate Cancer. JAMA, 271, 368-374. http://dx.doi.org/10.1001/jama.1994.03510290050036

[6] Magi-Galluzzi, C., Luo, J., Isaacs, W.B., Hicks, J.L., de Marzo, A.M. and Epstein, J.I. (2003) Alpha-Methylacyl-CoA Racemase: A Variably Sensitive Immunohistochemical Marker for the Diagnosis of Small Prostate Cancer Foci on Needle Biopsy. The American Journal of Surgical Pathology, 27, 1128-1133. http://dx.doi.org/10.1097/00000478-200308000-00010

[7] Heidenreich, A., et al. (2008) EAU Guidelines on Prostate Cancer. European Urology, 53, 68-80. http://dx.doi.org/10.1016/j.eururo.2007.09.002

[8] Epstein, J.I. and Y. XJ. (2002) Prostate Biopsy Interpretation. 3rd Edition, 320p.

[9] Algaba, F., et al. (1996) Assessment of Prostate Carcinoma in Core Needle Biopsy-Definition of Minimal Criteria for the Diagnosis of Cancer in Biopsy Material. Cancer, 78, 376-381.

[10] Thorson, P. and Humphrey, P.A. (2000) Minimal Adenocarcinoma in Prostate Needle Biopsy Tissue. American Journal of Clinical Pathology, 114, 896-909. http://dx.doi.org/10.1309/KVPX-C1EM-142L-1M6W

[11] Iczkowski, K.A., Chen, H.M., Yang, X.J. and Beach, R.A. (2002) Prostate Cancer Diagnosed after Initial Biopsy with Atypical Small Acinar Proliferation Suspicious for Malignancy Is Similar to Cancer Found on Initial Biopsy. Urology, 60, 851-854. http://dx.doi.org/10.1016/S0090-4295(02)01981-7

[12] Molinie, V., Hervé, J.M., Lugagne, P.M., Lebret, T. and Botto, H. (2006) Diagnostic Utility of a p63/Alpha-Methyl Coenzyme A Racemase (p504s) Cocktail in Ambiguous Lesions of the Prostate upon Needle Biopsy. BJU International, 97, 1109-1115. http://dx.doi.org/10.1111/j.1464-410X.2006.06069.x

[13] Shah, R.B., Zhou, M., LeBlanc, M., Snyder, M. and Rubin, M.A. (2002) Comparison of the Basal Cell-Specific Markers, 34betaE12 and p63, in the Diagnosis of Prostate Cancer. The American Journal of Surgical Pathology, 26, 11611168. http://dx.doi.org/10.1111/j.1464-410X.2006.06069.x

[14] Srigley, J.R. (2004) Benign Mimickers of Prostatic Adenocarcinoma. Modern Pathology, 17, 328-348. http://dx.doi.org/10.1038/modpathol.3800055

[15] Troxel, D.B. and Sabella, J.D. (1994) Problem Areas in Pathology Practice. Uncovered by a Review of Malpractice Claims. The American Journal of Surgical Pathology, 18, 821-831. http://dx.doi.org/10.1097/00000478-199408000-00009

[16] Jiang, Z., Woda, B.A., Wu, C.L. and Yang, X.J. (2004) Discovery and Clinical Application of a Novel Prostate Cancer Marker: Alpha-Methylacyl CoA Racemase (P504S). American Journal of Clinical Pathology, 122, 275-289. http://dx.doi.org/10.1309/EJUYUQPEX1MG68MK

[17] Brawer, M.K., Peehl, D.M., Stamey, T.A. and Bostwick, D.G. (1985) Keratin Immunoreactivity in the Benign and Neoplastic Human Prostate. Cancer Research, 45, 3663-3667.

[18] Ouyang, B., et al. (2011) Alpha-Methylacyl-CoA Racemase Spliced Variants and Their Expression in Normal and Malignant Prostate Tissues. Urology, 77, 249 e1-7.

[19] Gown, A.M. and Vogel, A.M. (1984) Monoclonal Antibodies to Human Intermediate Filament Proteins. II. Distribution of Filament Proteins in Normal Human Tissues. American Journal of Pathology, 114, 309-321.

[20] Henneberry, J.M., Kahane, H., Humphrey, P.A., Keetch, D.W. and Epstein, J.I. (1997) The Significance of Intraluminal Crystalloids in Benign Prostatic Glands on Needle Biopsy. The American Journal of Surgical Pathology, 21, 725728. http://dx.doi.org/10.1097/00000478-199706000-00014

[21] Wojno, K.J. and Epstein, J.I. (1995) The Utility of Basal Cell-Specific Anti-Cytokeratin Antibody (34 Beta E12) in the Diagnosis of Prostate Cancer. A review of 228 cases. The American Journal of Surgical Pathology, 19, 251-260. 
http://dx.doi.org/10.1097/00000478-199503000-00002

[22] Bostwick, D.G. and Brawer, M.K. (1987) Prostatic Intra-Epithelial Neoplasia and Early Invasion in Prostate Cancer. Cancer, 59, 788-794. http://dx.doi.org/10.1002/1097-0142(19870215)59:4<788::AID-CNCR2820590421>3.0.CO;2-I

[23] Signoretti, S., et al. (2000) p63 Is a Prostate Basal Cell Marker and Is Required for Prostate Development. American Journal of Pathology, 157, 1769-1775. http://dx.doi.org/10.1016/S0002-9440(10)64814-6

[24] Hedrick, L. and Epstein, J.I. (1989) Use of Keratin 903 as an Adjunct in the Diagnosis of Prostate Carcinoma. The American Journal of Surgical Pathology, 13, 389-396. http://dx.doi.org/10.1097/00000478-198905000-00006

[25] Martens, M.B. and Keller, J.H. (2006) Routine Immunohistochemical Staining for High-Molecular Weight Cytokeratin 34-Beta and Alpha-Methylacyl CoA Racemase (P504S) in Postirradiation Prostate Biopsies. Modern Pathology, 19, 287-290. http://dx.doi.org/10.1038/modpathol.3800531

[26] Rubin, M.A., Zhou, M., Dhanasekaran, S.M., Varambally, S., Barrette, T.R., Sanda, M.G., Pienta, K.J., Ghosh, D. and Chinnaiyan, A.M. (2002) $\alpha$-Methylacyl Coenzyme A Racemase as a Tissue Biomarker for Prostate Cancer. JAMA, 287, 1662-1670. http://dx.doi.org/10.1001/jama.287.13.1662

[27] Xu, J., Stolk, J.A., Zhang, X., Silva, S.J., Houghton, R.L., Matsumura, M., Vedvick, T.S., Leslie, K.B., Badaro, R. and Reed, S.G. (2000) Identification of Differentially Expressed Genes in Human Prostate Cancer Using Subtraction and Microarray. Cancer Research, 60, 1677-1682.

[28] Yang, X.J., Wu, C.L., Woda, B.A., Dresser, K., Tretiakova, M., Fanger, G.R. and Jiang, Z. (2002) Expression of $\alpha$ Methylacyl-CoA Racemase (P504S) in Atypical Adenomatous Hyperplasia of the Prostate. American Journal of Surgical Pathology, 26, 921-925. http://dx.doi.org/10.1097/00000478-200207000-00011

[29] Jiang, Z., Iczkowski, K.A., Woda, B.A., Tretiakova, M. and Yang, X.J. (2004) P504S Immunostaining Boosts Diagnostic Resolution of “Suspicious” Foci in Prostatic Needle Biopsy Specimens. American Journal of Clinical Pathology, 121, 99-107. http://dx.doi.org/10.1309/7T7RJCCL84JGXH3L

[30] Beach, R., Gown, A.M., De Peralta-Venturina, M.N., Folpe, A.L., Yaziji, H., Salles, P.G., Grignon, D.J., Fanger, G.R. and Amin, M.B. (2002) P504S Immunohistochemical Detection in 405 Prostatic Specimens Including 376 18-Gauge Needle Biopsies. American Journal of Surgical Pathology, 26, 1588-1596. http://dx.doi.org/10.1097/00000478-200212000-00006

[31] Kuefer, R., Varambally, S., Zhou, M., Lucas, P.C., Loeffler, M., Wolter, H., Mattfeldt, T., Hautmann, R.E., Gschwend, J.E., Barrette, T.R., Dunn, R.L., Chinnaiyan, A.M. and Rubin, M.A. (2002) $\alpha$-Methylacyl-CoA Racemase: Expression Levels of this Novel Cancer Biomarker Depend on Tumor Differentiation. American Journal of Pathology, 161, 841848. http://dx.doi.org/10.1016/S0002-9440(10)64244-7

[32] Zhou, M., Chinnaiyan, A.M., Kleer, C.G., Lucas, P.C. and Rubin, M.A. (2002) Alpha-Methylacyl-CoA Racemase: A Novel Tumor Marker Over-Expressed in Several Human Cancers and Their Precursor Lesions. American Journal of Surgical Pathology, 26, 926-931. http://dx.doi.org/10.1097/00000478-200207000-00012

[33] Luo, J., Zha, S., Gage, W.R., Dunn, T.A., Hicks, J.L., Bennett, C.J., Ewing, C.M., Platz, E.A., Ferdinandusse, S., Wanders, R.J., Trent, J.M., Isaacs, W.B. and De Marzo, A.M. (2002) Alpha-methylacyl-CoA Racemase: A New Molecular Marker for Prostate Cancer. Cancer Research, 62, 2220-2226.

[34] Zheng, S.L., Chang, B.L., Faith, D.A., Johnson, J.R., Isaacs, S.D., Hawkins, G.A., Turner, A., Wiley, K.E., Bleecker, E.R., Walsh, P.C., Meyers, D.A., Isaacs, W.B. and Xu, J. (2002) Sequence Variants of Alpha-methylacyl-CoA Racemase Are Associated with Prostate Cancer Risk. Cancer Research, 62, 6485-6488.

[35] Leav, I., McNeal, J.E., Ho, S.M. and Jiang, Z. (2003) $\alpha$-methylacyl-CoA Racemase (P504S) Expression in Evolving Carcinomas within Benign Prostatic Hyperplasia and in Cancers of the Transition Zone. Human Pathology, 34, 228-233. http://dx.doi.org/10.1053/hupa.2003.42

[36] Ferdinandusse, S., Denis, S., IJlst, L., Dacremont, G., Waterham, H.R. and Wanders, R.J. (2000) Subcellular Localization and Physiological Role of Alpha-methylacyl-CoA Racemase. Journal of Lipid Research, 41, 1890-1896.

[37] Aboseif, S., Shinohara, K., Weidner, N., Narayan, P. and Carroll, P.R. (1995) The Significance of Prostatic Intra-Epithelial Neoplasia. British Journal of Urology, 76, 355-359. http://dx.doi.org/10.1111/j.1464-410X.1995.tb07714.x

[38] Davidson, D., Bostwick, D.G., Qian, J., Wollan, P.C., Oesterling, J.E., Rudders, R.A., Siroky, M. and Stilmant, M. (1995) Prostatic Intraepithelial Neoplasia Is a Risk Factor for Adenocarcinoma: Predictive Accuracy in Needle Biopsies. Journal of Urology, 154, 1295-1299. http://dx.doi.org/10.1016/S0022-5347(01)66840-X

[39] Keetch, D.W., Humphrey, P., Stahl, D., Smith, D.S. and Catalona, W.J. (1995) Morphometric Analysis and Clinical Followup of Isolated Prostatic Intraepithelial Neoplasia in Needle Biopsy of the Prostate. Journal of Urology, 154, 347-351. http://dx.doi.org/10.1016/S0022-5347(01)67044-7

[40] Kronz, J.D., Allan, C.H., Shaikh, A.A. and Epstein, J.I. (2001) Predicting Cancer following a Diagnosis of High-Grade Prostatic Intraepithelial Neoplasia on Needle Biopsy: Data on Men with More than One Follow-Up Biopsy. American Journal of Surgical Pathology, 25, 1079-1085. http://dx.doi.org/10.1097/00000478-200108000-00014 
[41] O’Dowd, G.J., Miller, M.C., Orozco, R. and Veltri, R.W. (2000) Analysis of Repeated Biopsy Results within 1 Year after a Noncancer Diagnosis. Urology, 55, 553-559. http://dx.doi.org/10.1016/S0090-4295(00)00447-7

[42] Raviv, G., Janssen, T., Zlotta, A.R., Louis, L., Descamps, F., Verhest, A. and Schulman, C.C. (1996) High-Grade Intraepithelial Prostatic Neoplasms: Diagnosis and Association with Prostate Cancer. Acta Urologica Belgica, 64, 1115.

[43] Shepherd, D., Keetch, D.W., Humphrey, P.A., Smith, D.S. and Stahl, D. (1996) Repeat Biopsy Strategy in Men with Isolated Prostatic Intraepithelial Neoplasia on Prostate Needle Biopsy. Journal of Urology, 156, 460-463. http://dx.doi.org/10.1016/S0022-5347(01)65881-6

[44] Weinstein, M.H. and Epstein, J.I. (1993) Significance of High-Grade Prostatic Intraepithelial Neoplasia on Needle Biopsy. Human Pathology, 24, 624-629. http://dx.doi.org/10.1016/0046-8177(93)90242-9

[45] Zhou, M., Jiang, Z. and Epstein, J.I. (2003) Expression and Diagnostic Utility of Alpha-Methylacyl-CoA-Racemase (P504S) in Foamy Gland and Pseudohyperplastic Prostate Cancer. American Journal of Surgical Pathology, 27, 772 778. http://dx.doi.org/10.1097/00000478-200306000-00007

[46] Jiang, Z., Woda, B.A., Rock, K.L., Xu, Y., Savas, L., Khan, A., Pihan, G., Cai, F., Babcook, J.S., Rathanaswami, P., Reed, S.G., Xu, J. and Fanger, G.R. (2001) P504S: A New Molecular Marker for the Detection of Prostate Carcinoma. American Journal of Surgical Pathology, 25, 1397-1404. http://dx.doi.org/10.1097/00000478-200111000-00007

[47] Yang, X.J., Laven, B., Tretiakova, M., Blute Jr., R.D., Woda, B.A., Steinberg, G.D. and Jiang, Z. (2003) Detection of Alpha-Methylacyl-Coenzyme A Racemase in Postradiation Prostatic Adenocarcinoma. Urology, 62, 282-286. http://dx.doi.org/10.1016/S0090-4295(03)00259-0

[48] Zha, S., Ferdinandusse, S., Denis, S., Wanders, R.J., Ewing, C.M., Luo, J., De Marzo, A.M. and Isaacs, W.B. (2003) Alpha-Methylacyl-CoA Racemase as an Androgen-Independent Growth Modifier in Prostate Cancer. Cancer Research, 63, 7365-7376.

[49] He, L., Long, L.R., Antani, S. and Thoma, G.R. (2012) Histology Image Analysis for Carcinoma Detection and Grading. Computer Methods and Programs in Biomedicine, 107, 538-556. http://dx.doi.org/10.1016/j.cmpb.2011.12.007

[50] Diamond, J., Anderson, N.H., Bartels, P.H., Montironi, R. and Hamilton, P.W. (2004) The Use of Morphological Characteristics and Texture Analysis in the Identification of Tissue Composition in Prostatic Neoplasia. Human Pathology, 35, 1121-1131. http://dx.doi.org/10.1016/j.humpath.2004.05.010

[51] Stotzka, R., Männer, R., Bartels, P.H. and Thompson, D. (1995) A Hybrid Neural and Statistical Classifier System for Histopathologic Grading of Prostatic Lesions. Analytical and Quantitative Cytology and Histology, 17, $204-218$.

[52] Epstein, J.I., Allsbrook Jr., W.C., Amin, M.B., Egevad, L.L. and ISUP Grading Committee (2005) The 2005 International Society of Urological Pathology (ISUP) Consensus Conference on Gleason Grading of Prostatic Carcinoma. American Journal of Surgical Pathology, 29, 1228-1242. http://dx.doi.org/10.1097/01.pas.0000173646.99337.b1

[53] Rubin, M.A., Zerkowski, M.P., Camp, R.L., Kuefer, R., Hofer, M.D., Chinnaiyan, A.M. and Rimm, D.L. (2004) Quantitative Determination of Expression of the Prostate Cancer Protein Alpha-methylacyl-CoA Racemase Using Automated Quantitative Analysis (AQUA): A Novel Paradigm for Automated and Continuous Biomarker Measurements. American Journal of Pathology, 164, 831-840. http://dx.doi.org/10.1016/S0002-9440(10)63171-9

[54] Bailar III, J.C., Mellinger, G.T. and Gleason, D.F. (1966) Survival Rates of Patients with Prostatic Cancer, Tumor Stage, and Differentiation-Preliminary Report. Cancer Chemotherapy Reports, 50, 129-136.

[55] Gleason, D.F. (1966) Classification of Prostatic Carcinomas. Cancer Chemotherapy Reports, 50, 125-128.

[56] Gleason, D.F. and Mellinger, G.T. (1974) Prediction of Prognosis for Prostatic Adenocarcinoma by Combined Histological Grading and Clinical Staging. Journal of Urology, 111, 58-64.

[57] Jiang, Z., Wu, C.L., Woda, B.A., Dresser, K., Xu, J., Fanger, G.R. and Yang, X.J. (2002) P504S/ $\alpha$-Methylacyl-CoA Racemase: A Useful Marker for Diagnosis of Small Foci of Prostatic Carcinoma on Needle Biopsy. American Journal of Surgical Pathology, 26, 1169-1174. http://dx.doi.org/10.1097/00000478-200209000-00007

[58] Lin, F., Brown, R.E., Shen, T., Yang, X.J. and Schuerch, C. (2004) Immunohistochemical Detection of P504S in Primary and Metastatic Renal Cell Carcinomas. Applied Immunohistochemistry \& Molecular Morphology, 12, 153-159. http://dx.doi.org/10.1097/00129039-200406000-00010

\section{Abbreviations}

AMACR: alpha-methylacyl-CoA-racemase; H\&E: hematoxylin and eosin; IHC: immunohistochemistry; PIN: prostatic intraepithelial neoplasia; PNB: prostatic core needle biopsy; PSA: prostate-specific antigen. 
Scientific Research Publishing (SCIRP) is one of the largest Open Access journal publishers. It is currently publishing more than 200 open access, online, peer-reviewed journals covering a wide range of academic disciplines. SCIRP serves the worldwide academic communities and contributes to the progress and application of science with its publication.

Other selected journals from SCIRP are listed as below. Submit your manuscript to us via either submit@scirp.org or Online Submission Portal.
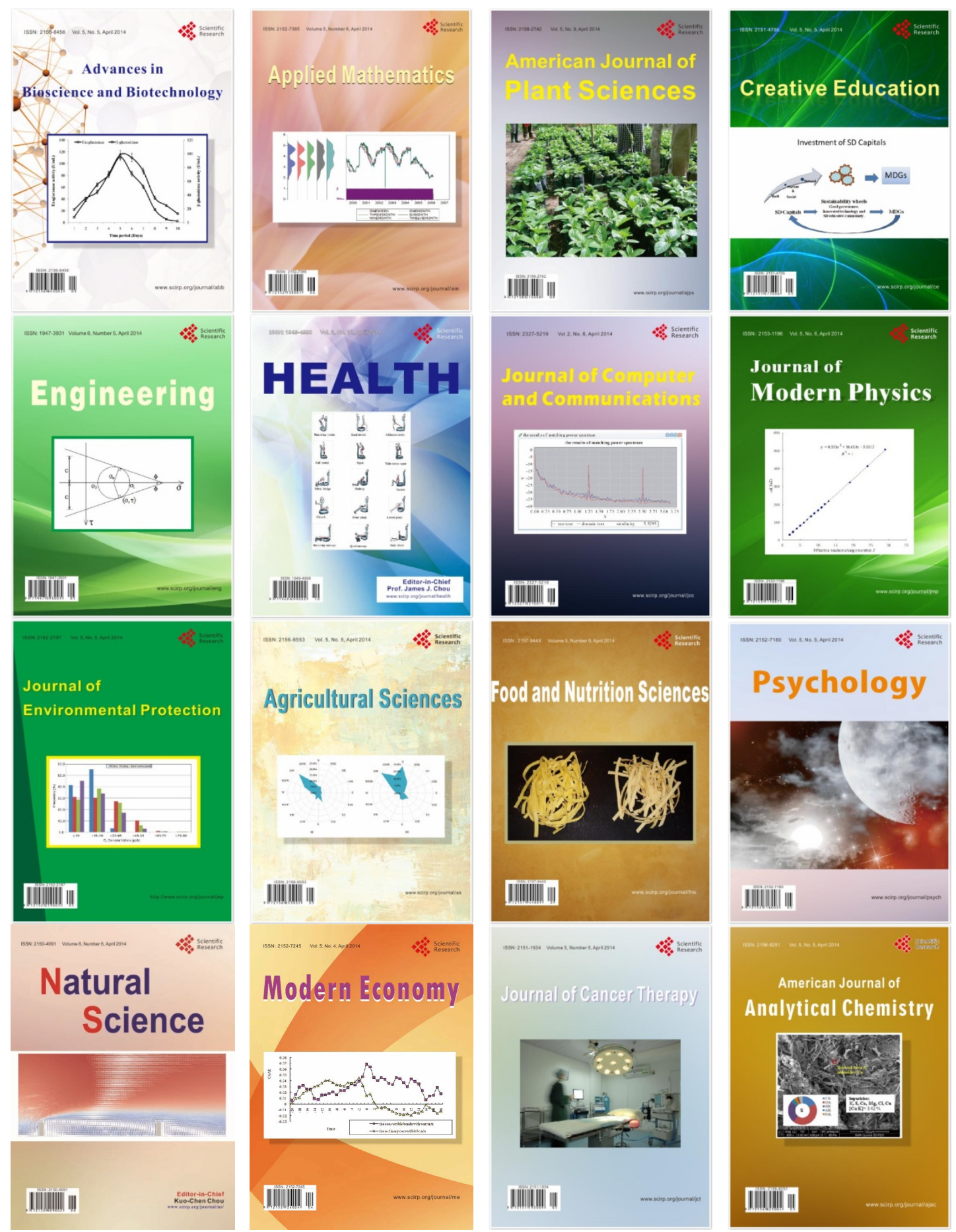